\title{
Inhibitory Effect of Dihydroartemisinin, An Active Ingredient of Artemisia annua, on Lipid Accumulation in Differentiating 3T3-L1 Preadipocytes
}

\author{
Byeong-Churl Jang \\ Department of Molecular Medicine, College of Medicine, Keimyung University
}

Received: April 2, 2020

Revised: May 4, 2020

Accepted: May 19, 2020

Correspondence to: Byeong-Churl Jang Department of Molecular Medicine, College of Medicine, Keimyung University, 1095, Dalgubeoldaero, Dalseo-gu, Daegu 42601, Korea

Tel: +82-53-254-7404

Fax: +82-53-254-7403

E-mail: lympia2012@naver.com, jangbc123@gw.kmu.ac.kr

Copyright $\odot 2020$ by The Society of Korean Medicine for Obesity Research

\begin{abstract}
Objectives: Artemisinin and its derivatives extracted from Artemisia annua, a Chinese herbal medicine, have variable biological effects due to structural differences. Up to date, the anti-obesity effect of dihydroartemisinin (DHA), a derivative of artemisinin, is unknown. The purpose of this study was to investigate the anti-adipogenic and lipolytic effects of DHA on 3T3-L1 preadipocytes.

Methods: Oil Red O staining and AdipoRed assay were used to measure lipid accumulation and triglyceride (TG) content in 3T3-L1 cells, respectively. Cell count analysis was used to determine the cytotoxicity of 3T3-L1 cells. Western blot and real-time reverse transcription polymerase chain reaction analyses were used to analyze the expression of protein and mRNA in 3T3-L1 cells, respectively.

Results: DHA at $5 \mu \mathrm{M}$ markedly inhibited lipid accumulation and reduced TG content in differentiating 3T3-L1 cells with no cytotoxicity. Furthermore, DHA at $5 \mu \mathrm{M}$ inhibited the expression of CCAAT/enhancer-binding protein- $\alpha(C / E B P-\alpha)$, peroxisome proliferator-activated receptor- $\gamma$ (PPAR $-\gamma$ ), fatty acid synthase (FAS), and perilipin A as well as the phosphorylation of signal transducer and activator of transcription-3 (STAT-3) in differentiating 3T3-L1 cells. Moreover, while DHA at $5 \mu \mathrm{M}$ had no effect on the mRNA expression of adiponectin, it strongly suppressed that of leptin in differentiating 3T3-L1 cells. However, DHA at $5 \mu \mathrm{M}$ had no lipolytic effect on differentiated 3T3-L1 cells, as assessed by no enhancement of glycerol release.

Conclusions: These results demonstrate that DHA at $5 \mu \mathrm{M}$ has a strong anti-adipogenic effect on differentiating 3T3-L1 cells through the reduced expression and phosphorylation of C/EBP- $\alpha$, PPAR $-\gamma$, FAS, perilipin A, and STAT-3.
\end{abstract}

Key Words: Dihydroartemisinin, C/EBP- $\alpha$, PPAR $-\gamma$, STAT-3, Perilipin A, 3T3-L1

\section{Introduction}

Obesity is defined as abnormal fat accumulation in the human body. Obesity has now become global pandemic, given that it causes the development of multiple human chronic diseases like type 2 diabetes, heart disease, hyperlipidemia, and cancer $^{1,2)}$. It is well documented that obesity is induced by excessive differentiation of adipocytes of the adipose tissue ${ }^{3)}$. Thus, any substance that suppresses excessive adipocyte differentiation, also called as adipogenesis, could have the potential to be a preventative and therapeutic agent for obesity.
Adipocyte differentiation is a biological multistep process that occurs in the form of cellular, morphological and biochemical changes ${ }^{4)}$. The process converts fibroblast-like preadipocytes into differentiated or mature adipocytes, which are characterized by sequential changes in the expression of specific genes that determine the specific adipocyte phenotype of the cells and by continuous accumulation of lipid droplets (LDs) that increases the hypertrophy of the cells ${ }^{3-5}$. There is a wealth of information that expression and phosphorylation of multiple adipogenic transcription factors, including CCAAT/enhancer-binding proteins (C/EBPs), perox- 
isome proliferator-activated receptors (PPARs), and signal transducer and activator of transcription (STAT) proteins, are crucial for preadipocyte differentiation ${ }^{6-8)}$. It is further shown that expression and activity of lipogenesis and lipid uptake (storage)-related enzymes, such as fatty acid synthase (FAS) and lipoprotein lipase (LPL), and LD associated proteins like perilipin A are also necessary for preadipocyte differentiation ${ }^{9-13)}$. Lipolysis, which is currently considered more important as a treatment for obesity, is the metabolic pathway through which triglyceride (TG) is hydrolyzed into a glycerol and three fatty acids ${ }^{14)}$. It is shown that a number of enzymes and signing factors, including hormone-sensitive lipase (HSL), protein kinase A (PKA), and extracellular signal-regulated kinase-1/2 (ERK-1/2), involve in the control of lipolysis in differentiated adipocytes ${ }^{14,15)}$.

Dihydroartemisinin (DHA) is the primary active derivative of artemisinin extracted from Artemisia annua, a Chinese herbal medicine ${ }^{16)}$ and has been shown to have anti-malarial, anti-inflammatory, anti-oxidative, browning, and anti-tumor effects ${ }^{17-21)}$. I have previously reported that artesunate, another artemisinin derivative, inhibits adipogenesis in 3T3-L1 preadipocytes ${ }^{22)}$. Up to date, the anti-obesity effect and mechanism of DHA that is structurally different to artesunate are not known. This led me to hypothesize that DHA might have similar or superior anti-adipogenic effects than artesunate. Therefore, in this study, the effects of DHA on lipid accumulation and lipolysis in differentiating and differentiated 3T3-L1 cells, a murine white preadipocyte, were investigated.

\section{Materials and Methods}

\section{Materials}

DHA was bought from Selleckchem (Houston, TX, USA). Primary antibodies of STAT-3, phospho (p)-STAT-3, STAT-5, p-STAT-5, C/EBP- $\alpha$, and PPAR- $\gamma$ were obtained from Santa Cruz Biotechnology (Delaware, CA, USA). Primary FAS antibody was bought from BD Bioscience (San Jose, CA, USA). Primary antibodies of perilipin A and $\beta$-actin were obtained from Bio Vision (Milpitas, CA, USA) and Sigma (St. Louis,
MO, USA), respectively. Enhanced chemiluminescence (ECL) reagent was bought from Advansta (Menlo Park, CA, USA). 3-isobutyl-1-methylxanthine (IBMX), dexamethasone, and insulin were purchased from Sigma.

\section{Differentiation of 3T3-L1 preadipocytes}

3T3-L1 murine white preadipocytes (ATCC, Manassas, VA, USA) were grown up to the contact inhibition stage and remained in the post-confluent stage for 2 days in Dulbecco's Modified Eagle's medium (DMEM) supplemented with 10\% calf bovine serum (Gibco, Long Islands, NY, USA) and penicillin-streptomycin (Welgene, Daegu, Korea). Differentiation was induced by changing the medium to DMEM supplemented with $10 \%$ fetal bovine serum (FBS; Welgene) plus a cocktail of hormones (MDI) that include $0.5 \mathrm{mM}$ IBMX (M) (Sigma), $0.5 \mu \mathrm{M}$ dexamethasone (D) (Sigma), and $5 \mu \mathrm{g} / \mathrm{mL}$ insulin (Sigma) in the absence or presence of DHA at the indicated concentrations or $5 \mu \mathrm{M}$. After $48 \mathrm{~h}$ MDI-induction, the differentiation medium was replaced with DMEM supplemented with $10 \%$ FBS and $5 \mu \mathrm{g} / \mathrm{mL}$ insulin in the absence or presence of DHA at the indicated concentrations or $5 \mu \mathrm{M}$ for additional 3 days. The cells were then fed every other day with DMEM containing $10 \%$ FBS in the absence or presence of DHA at the indicated concentrations or $5 \mu \mathrm{M}$ for additional 3 days.

\section{Oil Red O staining}

On day 8 of differentiation, control or DHA-treated 3T3-L1 cells were washed twice with PBS, fixed with $10 \%$ formaldehyde for $2 \mathrm{~h}$ at room temperature (RT), washed with $60 \%$ isopropanol and dried completely. The fixed cells were then stained with Oil Red $\mathrm{O}$ working solution for $1 \mathrm{~h}$ at RT and then washed twice with distilled water. LDs accumulated in control or DHA-treated 3T3-L1 cells were observed by light microscopy (TS100; Nikon, Tokyo, Japan).

\section{Cell count analysis}

3T3-L1 preadipocytes were seeded in 24-well plates and incubated overnight. Cells were similarly grown under the above-mentioned differentiation conditions. On day 8 of dif- 
ferentiation, control or DHA-treated 3T3-L1 cells, which cannot be stained with trypan blue dye, was counted under microscope. The cell count assay was done in triplicates. Data are mean \pm standard error (SE) of three independent experiments.

\section{Quantification of cellular TG content by AdipoRed assay}

On day 8 of differentiation, intracellular TG content in control or DHA-treated 3T3-L1 cells was measured using a commercially available AdipoRed Assay Reagent kit according to the manufacturer's instructions (Lonza, Basel, Switzerland). After a $10 \mathrm{~min}$ incubation, fluorescence was measured on Victor3 (Perkin Elmer Inc., Waltham, MA, USA) with an excitation at $485 \mathrm{~nm}$ and an emission at $572 \mathrm{~nm}$.

\section{Preparation of whole cell lysates}

At the designated time point, 3T3-L1 cells were washed twice with PBS and exposed to a modified radioimmuno precipitation assay buffer (50 mM Tris- $\mathrm{Cl}$ [pH 7.4], 150 $\mathrm{mM} \mathrm{NaCl}, 0.1 \%$ sodium dodecyl sulfate, $0.25 \%$ sodium deoxycholate, $1 \%$ Triton X-100, 1\% Nonidet P-40, 1 mM ethylenediaminetetraacetic acid, $1 \mathrm{mM}$ ethylene glycol tetraacetic acid, proteinase inhibitor cocktail [1x]). The cell lysates were then collected and centrifuged at 12,000 rpm for $20 \mathrm{~min}$ at $4^{\circ} \mathrm{C}$. The supernatant was saved, and protein concentrations were determined with pierce BCA protein assay kit (Thermo Scientific, Rockford, IL, USA).

\section{Western blot analysis}

Proteins $(50 \mu \mathrm{g})$ were separated by sodium dodecyl sulfate-polyacrylamide gel electrophoresis (10\%) and transferred onto nitrocellulose membranes (Millipore, Burlington, MA, USA). The membranes were washed with TBS (10 mM Tris, $150 \mathrm{mM} \mathrm{NaCl}$ ) supplemented with $0.05 \%$ (vol/vol) Tween 20 (TBST) followed by blocking with TBST containing $5 \%(\mathrm{w} / \mathrm{v})$ non-fat dried milk. The membranes were incubated overnight with antibodies specific for $\mathrm{C} / \mathrm{EBP}-\alpha(1: 1,000)$, PPAR- $\beta$ (1:1,000), PPAR- $\gamma(1: 1,000)$, p-STAT-3 $(1: 2,000)$, STAT-3 (1:2,000), p-STAT-5 (1:2,000), STAT-5 (1:2,000), FAS (1:1,000), perilipin $\mathrm{A}(1: 2,000)$ or $\beta$-actin $(1: 10,000)$ at $4^{\circ} \mathrm{C}$. The membranes were then exposed to secondary antibodies coupled to horseradish peroxidase for $2 \mathrm{~h}$ at RT. The membranes were washed three times with TBST at RT. Immunoreactivities were detected by ECL reagents. Equal protein loading was assessed by the expression level of actin protein.

\section{Reverse transcriptase-polymerase chain reaction (RT-PCR) analysis}

Total cellular RNA in the control or DHA-treated 3T3-L1 cells was isolated with the RNAiso Plus (Takara Bio Inc., Kusatsu, Japan). Three micrograms of total RNA were reverse transcribed using a random hexadeoxynucleotide primer and reverse transcriptase. Single stranded cDNA was amplified by PCR with the following primers: C/EBP- $\alpha$ sense 5'-TTA CAACAGGCCAGGTTTCC-3'; antisense 5'-CTCTGGGATGG ATCGATTGT-3'; PPAR- $\gamma$ sense 5'-GGTGAAACTCTGGG AGATTC-3'; antisense 5'-CAACCATTGGGTCAGCTCTC-3'; FAS sense 5'-TTGCTGGCACTACAGAATGC-3'; antisense 5'-AACAGCCTCAGAGCGACAAT-3'; perilipin A sense 5'CTTTCTCGACACACCATGGAAACC-3'; antisense 5'-CC ACGTTATCCGTAACACCCTTCA-3'; LPL sense 5'-TGCCG CTGTTTTGTTTTACC-3'; antisense 5'-TCACAGTTTCTGC TCCCAGC-3'; adiponectin sense 5'-GGAGATGCAGGTCT TCTTGGT-3'; antisense 5'-TCCTGATACTGGTCGTAGGT GAA-3'; leptin sense 5'-CCAAAACCCTCATCAAGACC-3'; antisense 5'-CTCAAAGCCACCACCTCTGT-3'; actin sense 5'-GGTGAAGGTCGGTGTGAACG-3'; antisense 5'-GGTA GGAACACGGAAGGCCA-3'. Expression levels of actin mRNA expression were used as an internal control as well as loading control.

\section{Statistical analysis}

Cell count analysis was done in triplicates and repeated three times. Data were expressed as mean $\pm \mathrm{SE}$. The significance of difference was determined by one-way ANOVA. All significance testing was based upon a $\mathrm{P}$ value of $<0.05$. 


\section{Results}

\section{DHA dose-dependently inhibits lipid accumu- lation and reduces TG content in differentiating 3T3-L1 cells with no cytotoxicity}

Fig. 1A is the experimental protocol of 3T3-L1 preadipocyte differentiation. Initially, the treatment effect of various concentrations of DHA (Fig. 1B) on cell growth (survival) during the differentiation of 3T3-L1 preadipocytes into adipocytes for 8 days was investigated using cell count analysis. Treatment with DHA at the concentrations tested did not affect the survival of 3T3-L1 cells (Fig. 1C). Next, the treatment effect of various concentrations of DHA on intracellular TG content in 3T3-L1 cells on day 8 (D8) of differ- entiation was measured using an AdipoRed assay. As shown in Fig. 1D, treatment with DHA also reduced intracellular TG content on D8 of 3T3-L1 preadipocyte differentiation in a concentration-dependent manner. DHA regulation of lipid accumulation during the differentiation of 3T3-L1 preadipocytes into adipocytes for 8 days was further examined using Oil Red O staining. As shown in Fig. 1E (upper panels), many intracellular LDs were formed and accumulated in 3T3-L1 cells on D8 of differentiation, compared with undifferentiated cells on D0. However, treatment with DHA dose-dependently suppressed accumulation of LDs in differentiating 3T3-L1 cells on D8. The DHA's suppressive effect on accumulation of LDs in differentiating 3T3-L1 cells on D8 was also confirmed by light microscopy (Fig. 1E, lower

(A)

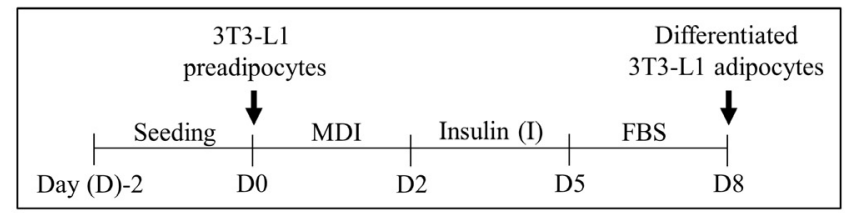

(B)

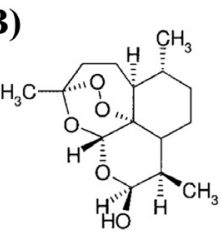

3T3-L1 preadipocytes (D0)
(C)

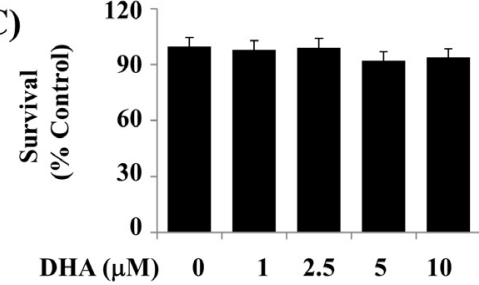

(D)

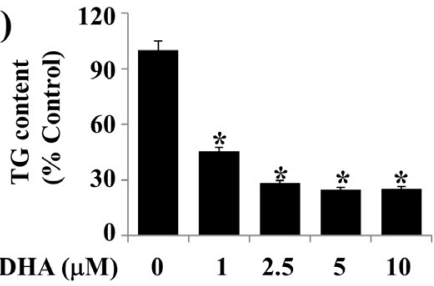

(E)
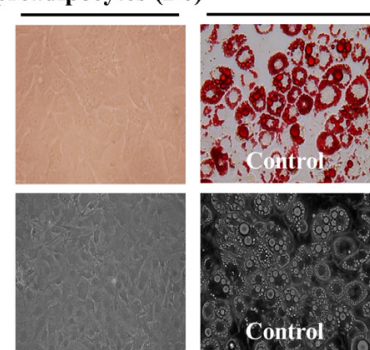

Differentiated

3T3-L1 adipocytes (D8)

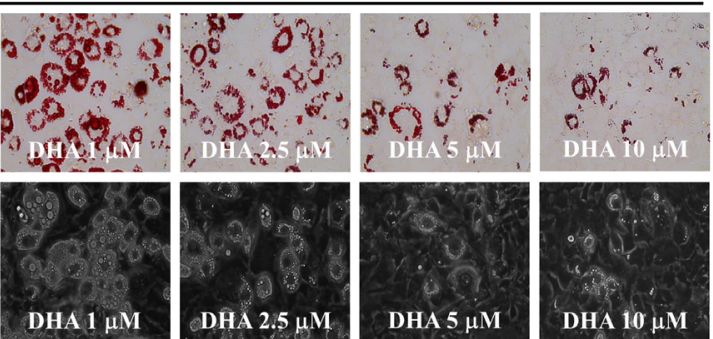

Fig. 1. Effects of DHA on lipid accumulation, TG content, and cell growth (survival) during 3T3-L1 preadipocyte differentiation. (A) Scheme for 3T3-L1 preadipocyte differentiation. (B) is the chemical structure of DHA. (C) Measurement of the number of surviving cells in vehicle control (DMSO 0.1\%) or DHA-treated 3T3-L1 preadipocytes on D8 by trypan blue dye exclusion. Data are mean \pm SE of three independent experiments, each done in triplicate. (D) Quantification of the intracellular TG content in vehicle control or DHA-treated 3T3-L1 preadipocytes on D8 by AdipoRed assay. Data are mean \pm SE of three independent experiments, each done in triplicate. ${ }^{*} \mathrm{P}<0.001$ vs. control (no DHA). (E) Measurement of the intracellular LDs accumulation in 3T3-L1 preadipocytes or differentiated adipocytes on D8 of differentiation in the absence (vehicle control; DMSO, 0.1\%) or presence of DHA at the indicated concentrations by Oil Red $\mathrm{O}$ staining. Phase-contrast images were also taken after the treatment (lower panels in E). MDI: 3-isobutyl-1-methylxanthine (M), dexamethasone (D), insulin (I), FBS: fetal bovine serum, DHA: dihydroartemisinin, TG: triglyceride, DMSO: dimethyl sulfoxide, D8: day 8, SE: standard error, LDs: lipid droplets. 
panels). Due to the maximal repressive effects on intracellular accumulation of LDs and TG content without cytotoxicity, this $5 \mu \mathrm{M}$ dose of DHA was chosen in further studies.

\section{DHA at $5 \mu \mathrm{M}$ strongly lowers expression and phosphorylation levels of C/EBP- $\alpha$, PPAR $-\gamma$, and STAT-3 in differentiating 3T3-L1 cells}

Next, the treatment effect of DHA at $5 \mu \mathrm{M}$ on protein expression and activity (phosphorylation) of adipogenesis-related transcription factors (C/EBP- $\alpha$, PPAR- $\gamma$, STAT-3, and STAT-5) was determined by Western blotting analysis. As shown in Fig. 2A, DHA at $5 \mu \mathrm{M}$ strongly reduced expression levels of $\mathrm{C} / \mathrm{EBP}-\alpha$ protein in differentiating 3T3-L1 cells on D5 and D8. Moreover, expression levels of PPAR- $\gamma$ protein in differentiating 3T3-L1 cells on D8 were strongly down-regulated by DHA at $5 \mu \mathrm{M}$. In addition, while DHA at $5 \mu \mathrm{M}$ did not affect phosphorylation levels of STAT-3 protein in differentiating 3T3-L1 cells on D2, it largely decreased those of STAT-3 protein on D5 and D8. However, DHA at $5 \mu \mathrm{M}$ had no or little effect on phosphorylation levels of STAT-5 protein in differentiating 3T3-L1 cells on D2, D5, and D8. Total protein expression levels of actin, STAT-3, and STAT-5 remained largely unchanged under these experimental conditions. RT-PCR analysis was next carried out to see whether DHA at $5 \mu \mathrm{M}$ affects mRNA expression levels of C/EBP- $\alpha$ and PPAR- $\gamma$ in differentiating 3T3-L1 cells. As shown in Fig. $2 \mathrm{~B}$, DHA at $5 \mu \mathrm{M}$ largely reduced $\mathrm{C} / \mathrm{EBP}-\alpha$ and PPAR- $\gamma$ transcripts in differentiating 3T3-L1 cells on D5 and D8. Expression levels of control actin mRNA remained constant under these experimental conditions.

\section{DHA at $5 \mu \mathrm{M}$ markedly reduces expression levels of FAS, perilipin $A$, and leptin in differentiating 3T3-L1 cells}

The treatment effect of DHA at $5 \mu \mathrm{M}$ on protein and/or mRNA expression levels of lipogenesis and lipid uptake (storage)-related enzymes (FAS, LPL) and LD associated protein (perilipin A) during 3T3-L1 preadipocyte differentiation was further investigated. As shown in Fig. 3A, DHA at 5 $\mu \mathrm{M}$ greatly reduced protein expression levels of FAS in differentiating 3T3-L1 cells on D8. Moreover, DHA at $5 \mu \mathrm{M}$ was able to largely decrease expression levels of perilipin A protein on D5 and D8. Furthermore, as shown in Fig. 3B, DHA at $5 \mu \mathrm{M}$ largely down-regulated mRNA levels of both
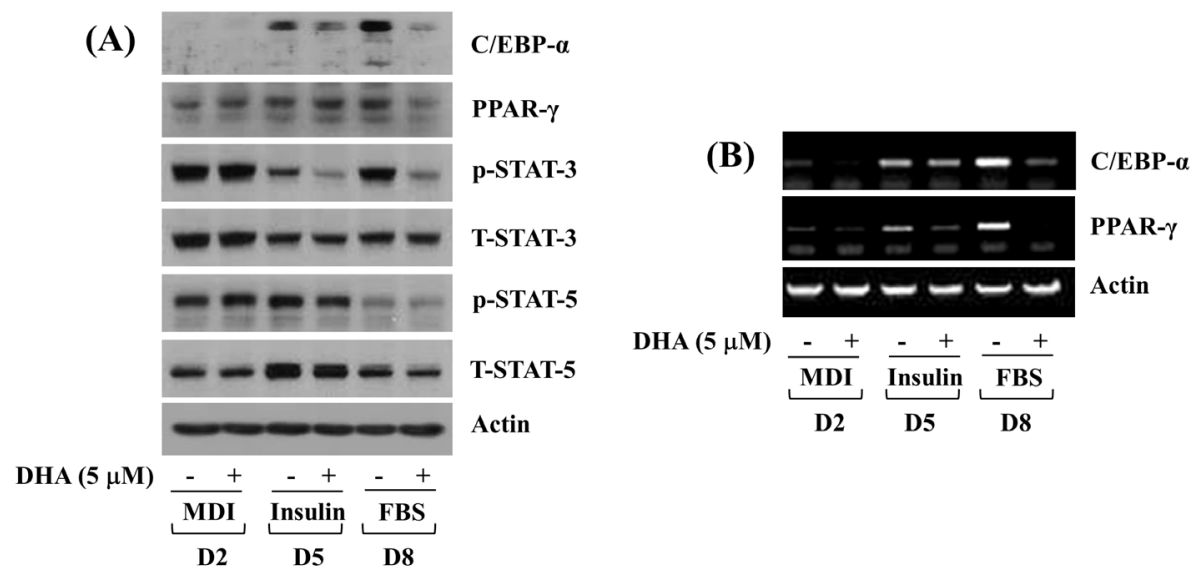

Fig. 2. Effects of DHA on expression and phosphorylation levels of $\mathrm{C} / \mathrm{EBP}-\alpha$, PPAR $-\gamma$, and STAT-3/5 during 3T3-L1 preadipocyte differentiation. (A) 3T3-L1 preadipocytes were differentiated with induction medium containing MDI, insulin, and FBS in the presence or absence of DHA, and harvested at days 2, 5, and 8, respectively. Whole cell lysates at the indicated time point were extracted and analyzed by Western blot analysis with respective antibodies. p-STAT-3, phosphorylated STAT-3; T-STAT-3, total STAT-3; p-STAT-5, phosphorylated STAT-5; T-STAT-5, total STAT-5. (B) 3T3-L1 preadipocytes were differentiated with induction medium containing MDI, insulin, and FBS in the presence or absence of DHA, and harvested at days 2, 5, and 8, respectively. Total cellular RNA at the indicated time point were extracted and analyzed by RT-PCR analysis with respective primers. DHA: dihydroartemisinin, C/EBP: CCAAT/enhancer-binding protein, PPAR: peroxisome proliferator-activated receptor, STAT: signal transducer and activator of transcription, MDI: 3-isobutyl-1-methylxanthine (M), dexamethasone (D), insulin (I), FBS: fetal bovine serum, D2: day 2, D5: day 5, D8: day 8, RT-PCR: reverse transcriptase-polymerase chain reaction. 
FAS and perilipin A in differentiating 3T3-L1 cells on D8. However, DHA at $5 \mu \mathrm{M}$ had no effect on LPL mRNA expression levels in differentiating 3T3-L1 cells on D2, D5, and D8. Notably, while DHA at $5 \mu \mathrm{M}$ did not influence adiponectin mRNA expression levels in differentiating 3T3-L1 cells on the times tested, it strongly reduced leptin expression levels on D5 and D8. Expression levels of control actin mRNA remained unchanged under these experimental conditions.

\section{DHA at $5 \mu \mathrm{M}$ has no lipolytic effect on differ- entiated 3T3-L1 cells}

Whether DHA at $5 \mu \mathrm{M}$ affects lipolysis in differentiated 3T3-L1 cells was next determined. DHA's lipolytic effect was herein assessed by measuring glycerol content in the

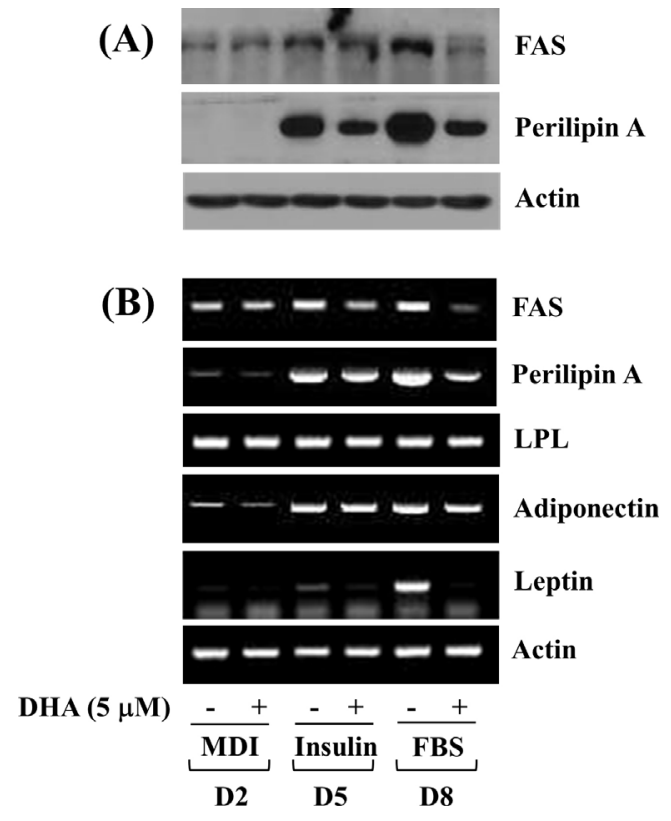

Fig. 3. Effects of DHA on expression levels of FAS, perilipin $A, L P L$, adiponectin, and leptin during 3T3-L1 preadipocyte differentiation. (A) 3T3-L1 preadipocytes were differentiated with induction medium containing $\mathrm{MDI}$, insulin, and FBS in the presence or absence of DHA, and harvested at days 2, 5, and 8, respectively. Whole cell lysates at the indicated time point were extracted and analyzed by Western blot analysis with respective antibodies. (B) 3T3-L1 preadipocytes were differentiated with induction medium containing $\mathrm{MDI}$, insulin, and FBS in the presence or absence of DHA, and harvested at days 2, 5, and 8, respectively. Total cellular RNA at the indicated time point were extracted and analyzed by RT-PCR analysis with respective primers. DHA: dihydroartemisinin, FAS: fatty acid synthase, LPL: lipoprotein lipase, MDI: 3-isobutyl-1-methylxanthine (M), dexamethasone (D), insulin (I), FBS: fetal bovine serum, D2: day 2, D5: day 5, D8: day 8, RT-PCR: reverse transcriptase-polymerase chain reaction. culture medium from differentiated 3T3-L1 cells that were serum starved for $2 \mathrm{~h}$ and treated without or with DHA for additional $3 \mathrm{~h}$, as depicted in Fig. 4A. Isoproterenol (ISO), a lipolytic agent ${ }^{23)}$, was included as a positive control. As expected, treatment with ISO at $20 \mu \mathrm{M}$ for $3 \mathrm{~h}$ greatly enhanced glycerol release in differentiated 3T3-L1 cells (Fig. 4B). However, DHA treatment at $5 \mu \mathrm{M}$ for $3 \mathrm{~h}$ led to a slight increase in glycerol content in differentiated 3T3-L1 cells. The treatment effects of DHA or ISO at the indicated dose on expression and phosphorylation levels of perilipin A and ERK-1/2, known lipolysis-related proteins, in differentiated 3T3-L1 cells were next compared. As shown in Fig. $4 \mathrm{C}$, while ISO treatment at $20 \mu \mathrm{M}$ for $3 \mathrm{~h}$ strongly down-regulated expression levels of perilipin A in differ-

(A)

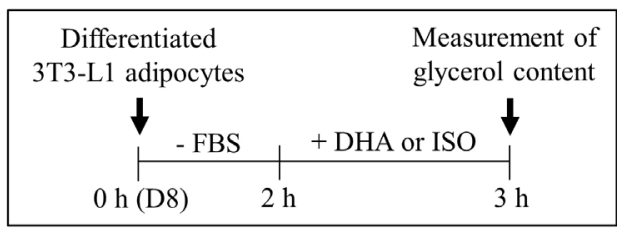

(B)
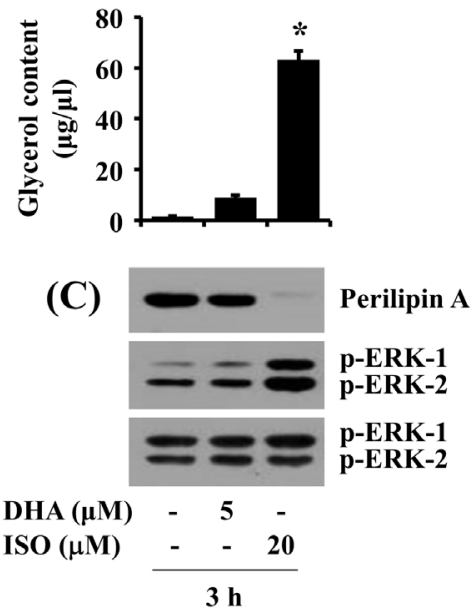

Fig. 4. Effects of DHA on lipolysis and expression and phosphorylation levels of perilipin A and ERK-1/2 in differentiated 3T3-L1 cells. (A) Scheme for measurement of glycerol content in differentiated 3T3-L1 adipocytes. (B) Differentiated 3T3-L1 cells on D8 were serum-starved for $2 \mathrm{~h}$ and treated with DHA or ISO for additional $3 \mathrm{~h}$. Glycerol content in culture medium from control or drug-treated cells was measured in triplicate. Data are mean $\pm S E$ of three independent experiments. ${ }^{*} \mathrm{P}<0.0024$ vs. control. (C) Differentiated 3T3-L1 cells on D8 were serum-starved for $2 \mathrm{~h}$ and treated with DHA or ISO for additional $3 \mathrm{~h}$. Whole cell lysates were extracted and analyzed by Western blot analysis with respective antibodies. FBS: fetal bovine serum, DHA: dihydroartemisinin, ISO: isoproterenol, D8: day 8, ERK: extracellular signal-regulated kinase, SE: standard error. 
entiated 3T3-L1 cells, DHA treatment at $5 \mu \mathrm{M}$ for $3 \mathrm{~h}$ had no or little effect on it. In addition, while treatment with ISO at $20 \mu \mathrm{M}$ for $3 \mathrm{~h}$ markedly increased phosphorylation levels of ERK-1/2 in differentiated 3T3-L1 cells, that with DHA at $5 \mu \mathrm{M}$ for $3 \mathrm{~h}$ did not. Total protein expression levels of ERK-1/2 remained largely unchanged under these experimental conditions.

\section{Discussion}

It is well recognized that excessive adipocyte differentiation and the resultant high lipid accumulation of the adipose tissue leads to the development of obesity. DHA, a derivative of artemisinin extracted from Artemisia annua ${ }^{16)}$, is reported to possess anti-malarial, anti-inflammatory, anti-oxidative, browning, and anti-tumor properties ${ }^{17-21)}$. Up to date, however, little is known about the anti-obesity effect and mode of action of DHA in white adipocytes. The present study demonstrates that DHA at $5 \mu \mathrm{M}$ has strong anti-adipogenic effect on differentiating 3T3-L1 white preadipocytes and the effect is controlled through modulation of the expression and phosphorylation levels of C/EBP- $\alpha$, PPAR- $\gamma$, FAS, perilipin A, and STAT-3.

Recently, it has been demonstrated that DHA at 5 or 7.5 $\mu \mathrm{M}$ induces browning-like features, as evidenced by cellular increase in multiple smaller LDs and mRNA expression levels of thermogenic markers (uncoupled protein 1 and peroxisome proliferator-activated receptor gamma co-activator 1 $\alpha$ ), in bone morphogenetic protein 4-commited C3H10T1/2 mouse mesenchymal stem cells ${ }^{20)}$, suggesting its browning effect. The present study displays that DHA at $5 \mu \mathrm{M}$ markedly reduces lipid accumulation and TG content in differentiating 3T3-L1 cells, advocating its anti-adipogenic and anti-lipogenic effects. Aforementioned, 3T3-L1 preadipocyte differentiation is largely influenced by the expression and activity of adipogenic transcription factors, including $\mathrm{C} / \mathrm{EBP}-\alpha$, PPAR- $\gamma$, and STAT-3/5 $5^{6,724-27)}$. It is further known that FAS, a lipogenic enzyme responsible for the synthesis of fatty acid $^{9)}$ and perilipin A, a protein that binds to and stabilizes the newly synthesized $\operatorname{LDs}^{9,11,13,14)}$ play important roles in lipid accumulation during 3T3-L1 preadipcyte differentiation. However, little is known about DHA regulation of C/EBP$\alpha$, PPAR- $\gamma$, STAT-3/5, FAS, and perilipin A during 3T3-L1 preadipcyte differentiation. In this study, DHA at $5 \mu \mathrm{M}$ greatly reduces expression and phosphorylation levels of C/EBP- $\alpha$, PPAR- $\gamma$, STAT-3, FAS, and perilipin A in differentiating 3T3-L1 cells. These findings suggest that DHA's anti-adipogenic and lipid-lowering effects on differentiating 3T3-L1 cells are likely to be due to the reduced expression and phosphorylation levels of C/EBP- $\alpha$, PPAR- $\gamma$, STAT-3, FAS, and perilipin A.

It is well known that the adipose tissue synthesizes and secretes an array of adipokines, including leptin and adiponectin $^{28,29)}$. Reportedly, while expression levels of adiponectin decrease with increase in the adiposity, those of lepin increase in obesity ${ }^{30-32)}$. It is further established that while adiponectin has many beneficial metabolic effects like reduction of serum glucose and lipids, maintenance of insulin sensitivity, and increase in fatty acid oxidation, leptin plays pathogenic roles in obesity and related disorders including insulin resistance ${ }^{32-35)}$. Consequently, decreased leptin expression is regarded an alternative against obesity and related disorders. In this study, it is shown that DHA at $5 \mu \mathrm{M}$ greatly reduces leptin, but not adiponectin, mRNA expression levels in differentiating 3T3-L1 cells, suggesting the potential utility of DHA for the prevention or treatment of obesity and related disorders associated with leptin overexpression.

Aforementioned, a number of enzymes and signaling proteins, including HSL, PKA, perilipin A, and ERK-1/2, participate in lipolysis in differentiated adipocytes ${ }^{13-15)}$. Among those, HSL is a major enzyme in the mobilization of fatty acids from stored TG. Accordingly, lipolysis is stimulated by activation of PKA which phosphorylates HSL on S563, S659, and S660 in adipocytes. Perilipin A is a highly expressed protein in both differentiating and differentiated adipocytes that are localized at the surface of LDs. It is believed that perilipin A has key roles in not only lipid packaging or storage (lipid accumulation) in differentiating preadipocytes but also lipid hydrolysis (lipolysis) in differentiated adipocytes. Supporting the latter, cellular loss (or 
down-regulation) of perilipin A facilitates the access of HSL into LDs in differentiated adipocytes, resulting in HSL mediated lipolysis ${ }^{36)}$. Increase in lipolysis through ERK-1/2-dependent HSL phosphorylation (on S600) and activation also has been reported ${ }^{15)}$. Given that while ISO largely enhances glycerol release and down-regulates perilipin A expression and up-regulates ERK-1/2 phosphorylation in differentiated 3T3-L1 cells, DHA does not have regulatory effects on them in this study, it is evident that DHA has no lipolytic effect on differentiated 3T3-L1 cells.

\section{Conclusion}

These results demonstrate firstly that DHA has a strong anti-adipogenic effect in differentiating 3T3-L1 preadipocytes and its anti-adipogenic effect is largely attributable to the reduced expression and phosphorylation levels of $\mathrm{C} / \mathrm{EBP}-\alpha$, PPAR- $\gamma$, FAS, perilipin A, and STAT-3. The findings presented herein suggest that DHA may be applied as a potential anti-obesity agent.

\section{Acknowledgements}

The author deeply thanks Mr. Anil Yadav Kumar for the correction of manuscript.

\section{References}

1. Kopelman PG. Obesity as a medical problem. Nature. $2000 ; 404(6778): 635-43$.

2. Abdelaal M, le Roux CW, Docherty NG. Morbidity and mortality associated with obesity. Ann Transl Med. 2017 ; $5(7): 161$.

3. Ali AT, Hochfeld WE, Myburgh R, Pepper MS. Adipocyte and adipogenesis. Eur J Cell Biol. 2013 ; 92(6-7) : 229-36.

4. Ghaben AL, Scherer PE. Adipogenesis and metabolic health. Nat Rev Mol Cell Biol. 2019 ; 20(4) : 242-58.

5. Onal G, Kutlu O, Gozuacik D, Dokmeci Emre S. Lipid droplets in health and disease. Lipids Health Dis. 2017 ; 16(1) : 128 .
6. Cao Z, Umek RM, McKnight SL. Regulated expression of three $\mathrm{C} / \mathrm{EBP}$ isoforms during adipose conversion of 3T3-L1 cells. Genes Dev. 1991 ; 5(9) : 1538-52.

7. Farmer SR. Transcriptional control of adipocyte formation. Cell Metab. 2006 ; 4(4) : 263-73.

8. Lehrke M, Lazar MA. The many faces of PPARgamma. Cell. 2005 ; 123(6) : 993-9.

9. Lakshmanan MR, Nepokroeff CM, Porter JW. Control of the synthesis of fatty-acid synthetase in rat liver by insulin, glucagon, and adenosine $3^{\prime}: 5^{\prime}$ cyclic monophosphate. Proc Natl Acad Sci USA. 1972 ; 69(12) : 3516-9.

10. Montalto MB, Bensadoun A. Lipoprotein lipase synthesis and secretion: effects of concentration and type of fatty acids in adipocyte cell culture. J Lipid Res. 1993 ; 34(3) : 397-407.

11. Beller M, Bulankina AV, Hsiao HH, Urlaub H, Jäckle H, Kühnlein RP. PERILIPIN-dependent control of lipid droplet structure and fat storage in drosophila. Cell Metab. 2010 ; 12(5) : 521-32.

12. Kern PA, Di Gregorio G, Lu T, Rassouli N, Ranganathan G. Perilipin expression in human adipose tissue is elevated with obesity. J Clin Endocrinol Metab. 2004 ; 89(3) : 1352-8.

13. Wolins NE, Brasaemle DL, Bickel PE. A proposed model of fat packaging by exchangeable lipid droplet proteins. FEBS Lett. 2006 ; 580(23) : 5484-91.

14. Duncan RE, Ahmadian M, Jaworski K, Sarkadi-Nagy E, Sul HS. Regulation of lipolysis in adipocytes. Annu Rev Nutr. 2007 ; 27 : 79-101.

15. Greenberg AS, Shen WJ, Muliro K, Patel S, Souza SC, Roth RA, et al. Stimulation of lipolysis and hormone-sensitive lipase via the extracellular signal-regulated kinase pathway. J Biol Chem. 2001 ; 276(48) : 45456-61.

16. Tu Y. Artemisinin-a gift from traditional chinese medicine to the world (nobel lecture). Angew Chem Int Ed Engl. 2016 ; 55(35) : 10210-26.

17. Sevene E, Banda CG, Mukaka M, Maculuve S, Macuacua S, Vala A, et al. Efficacy and safety of dihydroartemisinin-piperaquine for treatment of plasmodium falciparum uncomplicated malaria in adult patients on antiretroviral therapy in Malawi and Mozambique: an open label non-randomized interventional trial. Malar J. $2019 ; 18(1): 277$. 
18. Qu C, Ma J, Liu X, Xue Y, Zheng J, Liu L, et al. Dihydroartemisinin exerts anti-tumor activity by inducing mitochondrion and endoplasmic reticulum apoptosis and autophagic cell death in human glioblastoma cells. Front Cell Neurosci. $2017 ; 11$ : 310.

19. Liu X, Lu J, Liao Y, Liu S, Chen Y, He R, et al. Dihydroartemisinin attenuates lipopolysaccharide-induced acute kidney injury by inhibiting inflammation and oxidative stress. Biomed Pharmacother. $2019 ; 117$ : 109070.

20. Lu P, Zhang FC, Qian SW, Li X, Cui ZM, Dang YJ, et al. Artemisinin derivatives prevent obesity by inducing browning of WAT and enhancing BAT function. Cell Res. 2016 ; 26(10) : 1169-72.

21. Liu Y, Gao S, Zhu J, Zheng Y, Zhang H, Sun H. Dihydroartemisinin induces apoptosis and inhibits proliferation, migration, and invasion in epithelial ovarian cancer via inhibition of the hedgehog signaling pathway. Cancer Medicine. 2018 ; 7(11) : 5704-15.

22. Jang BC. Artesunate inhibits adipogeneis in 3T3-L1 preadipocytes by reducing the expression and/or phosphorylation levels of C/EBP- $\alpha$, PPAR- $\gamma$, FAS, perilipin A, and STAT-3. Biochem Biophys Res Commun. 2016; 474(1) : 220-5.

23. Zalatan F, Krause JA, Blask DE. Inhibition of isoproterenol-induced lipolysis in rat inguinal adipocytes in vitro by physiological melatonin via a receptor-mediated mechanism. Endocrinology. 2001 ; 142(9) : 3783-90.

24. Rosen ED, Sarraf P, Troy AE, Bradwin G, Moore K, Milstone DS, et al. PPAR $\gamma$ is required for the differentiation of adipose tissue in vivo and in vitro. Mol Cell. $1999 ; 4(4):$ 611-7.

25. Linhart HG, Ishimura-Oka K, DeMayo F, Kibe $\mathrm{T}$, Repka D, Poindexter B, et al. C/EBP $\alpha$ is required for differentiation of white, but not brown, adipose tissue. Proc Natl Acad Sci USA. 2001 ; 98(22) : 12532-7.

26. Stephens JM, Morrison RF, Pilch PF. The expression and regulation of STATs during 3T3-L1 adipocyte differentiation. J Biol Chem. 1996 ; 271(18) : 10441-4.

27. Rosen ED, Hsu CH, Wang X, Sakai S, Freeman MW, Gonzalez FJ, et al. C/EBP $\alpha$ induces adipogenesis through PPAR $\gamma$ : A unified pathway. Genes Dev. 2002 ; 16(1) : 22-6.

28. Kershaw EE, Flier JS. Adipose tissue as an endocrine organ. J Clin Endocrinol Metab. 2004 ; 89(6) : 2548-56.

29. Ahima RS. Adipose tissue as an endocrine organ. Obesity (Silver Spring). 2006 ; 14 Suppl 5 : 242S-9S.

30. Hwang CS, Loftus TM, Mandrup S, Lane MD. Adipocyte differentiation and leptin expression. Annu Rev Cell Dev Biol. 1997 ; 13 : 231-59.

31. Bastard JP, Maachi M, Lagathu C, Kim MJ, Caron M, Vidal H, et al. Recent advances in the relationship between obesity, inflammation, and insulin resistance. Eur Cytokine Netw. 2006 ; 17(1) : 4-12.

32. Ceddia RB, Somwar R, Maida A, Fang X, Bikopoulos G, Sweeney G. Globular adiponectin increases GLUT4 translocation and glucose uptake but reduces glycogen synthesis in rat skeletal muscle cells. Diabetologia. $2005 ; 48(1): 132-9$

33. Shehzad A, Iqbal W, Shehzad O, Lee YS. Adiponectin: regulation of its production and its role in human diseases. Hormones (Athens). $2012 ; 11(1)$ : 8-20.

34. Yadav A, Kataria MA, Saini V, Yadav A. Role of leptin and adiponectin in insulin resistance. Clin Chim Acta Int J Clin Chem. 2013 ; 417 : 80-4.

35. Friedman J. Fat in all the wrong places. Nature. 2002 ; 415(6869) : 268-9.

36. Miyoshi H, Souza SC, Zhang HH, Strissel KJ, Christoffolete MA, Kovsan J, et al. Perilipin promotes hormone-sensitive lipase mediated adipocyte lipolysis via phosphorylation-dependent and -independent mechanisms. J Biol Chem. 2006 ; 281(23) : 15837-44. 